\title{
miR-181a regulates the host immune response against Schistosoma japonicum infection through the TLR4 receptor pathway
}

Yixiao Tang ${ }^{1,2}$, Yuanxi Shen ${ }^{1,2}$, Yang Hong ${ }^{1,2^{*}}$, Zuhang Zhang ${ }^{1,2}$, Qi Zhai ${ }^{1,2}$, Zhiqiang Fu ${ }^{1,2}$, Hao Li ${ }^{1,2}$, Ke Lu $^{1,2}$ and Jiaojiao Lin ${ }^{1,2^{*}}$

\begin{abstract}
Background: Schistosomiasis japonica is a serious zoonotic parasitic disease. Preliminary studies have shown that the expression of microRNA-181a (miR-181a) in the liver, lung and spleen tissues of susceptible host BALB/c mice and resistant host reed vole (Microtus fortis) 10 days post-infection (dpi) with Schistosoma japonicum was significantly different from pre-infection levels. This difference suggests the possibility that miR-181a expression may be related to the regulation of the hosts' early immune response against S. japonicum infection and thereby affect the development and survival of parasites in their final hosts.
\end{abstract}

Methods: BALB/c mice, M. fortis, Toll-like receptor 4 (TLR4)-deficient mice and wild-type mice (C57BL/6) were infected with S. japonicum, and differences in miR-181a expression between BALB/c mice and M. fortis over different time points post-infection (0,3,7,10 and $14 \mathrm{dpi})$ were compared. MiR-181a mimic, miR-181a inhibitor and irrelevant miRNA, as well as lipopolysaccharide (LPS), a TLR4 receptor ligand, were used to transfect mouse RAW264.7 macrophages. The expression levels of the TLR4 pathway-related cytokines interleukin (IL)-1 $\beta$, tumor necrosis factor a (TNF-a) and IL-6 were detected by quantitative PCR analysis.

Results: The expression of miR-181a was significantly upregulated in the serum and liver of mice infected with $S$. japonicum and downregulated in the serum and liver of M. fortis. T-helper cell (Th1)-type cytokines, such as TNF-a, IL-6 and IL-1 $\beta$, and Th2-type cytokines, such as IL-10 and IL-4, were differentially expressed in M. fortis and BALB/c mice in the early stage of infection. The expression level of miR-181a in the serum was threefold higher in TLR4-deficient mice than in wild-type mice 10 dpi with S. japonicum. The expression of IL-1 $\beta$, TNF- $\alpha$ and IL-6 decreased in RAW264.7 cells transfected with miR-181a mimic and increased in cells transfected with miR-181a inhibitor. miR-181a expression was downregulated and the expressions of TLR4 and three TLR4 pathway-related cytokines (IL-1 $\beta$, IL-6, and TNF-a) were upregulated in RAW264.7 macrophages stimulated with the TLR4 receptor ligand LPS.

Conclusion: These results suggest the possibility of mutual regulation between miR-181a and the TLR4 signaling pathway during S. japonicum infection. miR-181a may regulate the expression of pro-inflammatory factors through the TLR4 receptor pathway and participate in the immunomodulatory effect of anti-S. japonicum infection.

\footnotetext{
*Correspondence: honyang_7@126.com; jjlin@shvri.ac.cn

${ }^{1}$ National Reference Laboratory for Animal Schistosomiasis, Shanghai

Veterinary Research Institute, Chinese Academy of Agricultural Sciences,

Shanghai 200241, P.R. China

Full list of author information is available at the end of the article
} original author(s) and the source, provide a link to the Creative Commons licence, and indicate if changes were made. The images or other third party material in this article are included in the article's Creative Commons licence, unless indicated otherwise in a credit line to the material. If material is not included in the article's Creative Commons licence and your intended use is not permitted by statutory regulation or exceeds the permitted use, you will need to obtain permission directly from the copyright holder. To view a copy of this licence, visit http://creativecommons.org/licenses/by/4.0/. The Creative Commons Public Domain Dedication waiver (http://creativeco mmons.org/publicdomain/zero/1.0/) applies to the data made available in this article, unless otherwise stated in a credit line to the data. 


\section{Graphical abstract:}

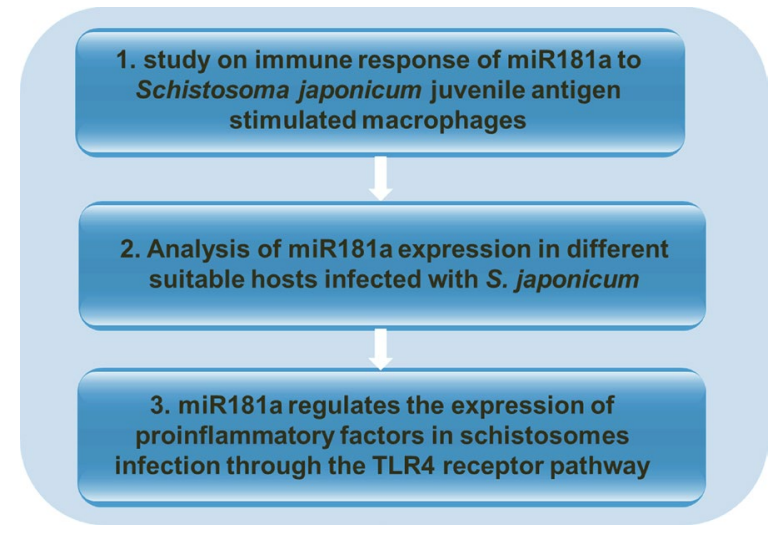

Keywords: Schistosoma japonicum, miR-181a, Microtus fortis, TLR4 receptor pathway, Cytokines

\section{Background}

Schistosomiasis is a tropical and subtropical parasitic disease caused by schistosome infection, with an estimated 200 million people infected and 800 million people living in schistosomiasis-endemic regions. Studies have shown that during the early period of schistosome infection in mice, the induction of a T-helper cell type I (Th1)-dominated immune response against schistosome infection is characterized by increased levels of interferon- $\gamma$ (IFN- $\gamma$ ) and tumor necrosis factor $\alpha$ (TNF- $\alpha)$, and that the host immune response shifts to a Th2-dominant immune response 4-6 weeks after infection which is characterized by increased expression levels of several interleukins (ILs), including IL-4, IL-13 and IL-12 [1]. Increased expression of these Th1- and Th2-associated cytokines is important in terms of host resistance to schistosome infection. Macrophages play a vital role in host resistance to schistosome infection and pathological changes in the liver. Macrophages can be activated into M1 macrophages and M2 macrophages. M1 macrophages are activated by the secretion of IFN- $\gamma$ (mainly secreted by $\mathrm{TH} 1$ cells, cytotoxic T cells and natural killer [NK] cells), lipopolysaccharide (LPS; a component of the outer membrane of Gram-negative bacteria) and granulocyte-macrophage colony-stimulating factor (GM-CSF) and mainly produce pro-inflammatory cytokines [2-4], such as TNF- $\alpha$, IL- $1 \beta$ and IL-12. M2 macrophages are activated by Th 2 cytokines and then play a regulatory role to maintain this chronic inflammatory disease. In contrast to M1 macrophages, M2 macrophages are involved in the Th2 bias response, producing transforming growth factor $\beta$ (TGF- $\beta$ ), IL-10, IL-13 and IL-4, which leads to parasite clearance and host protection [5-7].

The reed vole Microtus fortis is the only mammal host identified to date that exhibits resistance against schistosome infection. Studies have shown that $M$. fortis has a certain resistance to $S$. japonicum infection [8-11]. Such resistance can affect the growth and development of S. japonicum in the host, resulting in immature development of the schistosomes and the female not laying eggs, thereby protecting the host from severe disease [12]. Preliminary studies in our laboratory have also shown that 10 days after the non-susceptible host $M$. fortis and the susceptible host mice were infected with S. japonicum, expression of microRNA-181a (miR-181a) in liver, lung and spleen tissues was significantly different, possibly related to the regulation of host resistance to schistosome infection. An in-depth analysis of the differences in the expression of miR-181a and the effect of this differential expression of related cytokines in $M$. fortis and mice infected with schistosomes is highly relevant for elucidating the immune regulation mechanism of miR181a molecules in schistosome infection and the interaction mechanism between hosts and S. japonicum. In the present study, we collected liver tissue and serum samples from C57BL/6 mice and $M$. fortis infected with $S$. japonicum cercariae at different post-infection periods, and then analyzed and compared the expression levels of miR-181a in these two different suitable hosts after infection with $S$. japonicum. Our ultimate aim was to provide a basis for further understanding of the regulatory role of host miR-181a in the growth and development of schistosomes.

Toll-like receptors (TLRs) are a class of pathogenic pattern receptors that mediate innate immunity and are mainly involved in the recognition of molecular patterns associated with pathogenic microorganisms. It has been shown that transcription factors of signaling molecules 
can play a key role in regulating microRNA (miRNA) expression in the TLR signaling pathway by acting as transcriptional activators of miRNAs, which in turn initiate miRNA expression. Also, miRNAs have complex and subtle regulatory effects on the TLR signaling pathway, and inhibition of the TLR signaling pathway is crucial in suppressing excessive inflammatory responses [13]. These two processes regulate each other and interact with each other, thus forming a complex regulatory network.

TLR4 was first identified in 1997 with elevated expression in the inflammatory response [14]. It was the first TLR-related protein to be identified in humans and was subsequently found to be distributed in almost all cell lines, but mostly expressed in cells involved in host immune and defense functions, such as monocytes, lymphocytes, dendritic cells, granulocytes, epithelial cells, as well as more recently in renal tubular epithelial cells, heart respiratory epithelial cells and intestinal epithelial cells $[15,16]$. TLR4 can recognize and transmit signals by recognizing specific antigenic components of pathogen-associated molecular patterns. LPS is an important TLR4 ligand [17], and in response to LPS stimulation, it causes impaired activation of downstream cytokines [18]. Studies on humans have shown that LPS-mediated cytokine release is reduced in surgical patients, indicating a reduction in the innate immunity of cells to inflammation, which is not conducive to the recovery of the body [19]. The TLR4 signaling pathway is one of the more important inflammatory pathways as well as a relatively well-studied one, and this pathway is closely associated with the occurrence and development of many diseases. The expression of downstream inflammatory factors associated with TLR4 often affects the developmental direction of the disease and the post-cure situation. The relationship between the TLR pathway and miRNAs is crucial in terms of the maintenance of normal physiological functions in the body and the prevention of excessive inflammation and disease development. Moreover, study of this relationship provides a strategy for gaining a better understanding of diseases associated with the TLR signaling pathway. More specifically, exploring the relationship between the two is vital for furthering our understanding of host resistance to schistosome infection.

\section{Methods}

\section{Schistosome infection and sample preparation}

Six-week-old male BALB/c mice were purchased from Shanghai Slac Laboratory Animal Co., Ltd. (Shanghai, P.R. China) and the reed voles ( $M$. fortis) (each weighing about $60 \mathrm{~g}$ ) were kindly provided by the Shanghai Experimental Animal Center (Shanghai, P.R. China). Ten
BALB/c mice and ten $M$. fortis were each infected with $40 \mathrm{~S}$. japonicum cercariae via the abdomen. Blood samples were taken from the orbit on $0,3,7,10$ and 14 days post-infection (dpi). Subsequently, serum and liver samples were collected, and miRNA was extracted to measure the expression of miR-181a in mice and M. fortis at different time periods.

TLR4-deficient mice and C57BL/6 wild-type mice (WT; control group) were purchased from Nanjing Biomedical Research Institute of Nanjing University (Gulou, Nanjing, P.R. China). Five TLR4-deficient mice and five WT mice were infected with $40 \mathrm{~S}$. japonicum cercariae via the abdominal skin patch method, and serum was collected by dissection at $10 \mathrm{dpi}$ and stored at $-80^{\circ} \mathrm{C}$ for later use. The $S$. japonicum cercariae were provided by Shanghai Veterinary Research Institute, Chinese Academy of Agricultural Sciences (Shanghai, P.R. China).

\section{Culture and stimulation of RAW264.7 macrophages}

RAW264.7 macrophages were purchased from Applied Biological Materials Inc. (Richmond, BC, Canada), maintained at the Shanghai Veterinary Research Institute, Chinese Academy of Agricultural Sciences (Shanghai, P.R. China) and cultured in Dulbecco's Modified Eagle's Medium (DMEM; Invitrogen, Thermo Fisher Scientific, Waltham, MA, USA) supplemented with $10 \%$ heatinactivated fetal bovine serum (FBS; Invitrogen, Thermo Fisher Scientific) at $37^{\circ} \mathrm{C}, 5 \% \mathrm{CO}_{2}$ in an incubator. Cells were cultured into a 12-well culture plate at a density of $2 \times 10^{5}$ cells/well. When the cells were spread over $70 \%$ of the bottom, they were first stimulated with $20 \mu \mathrm{g} / \mathrm{ml}$ LPS for $12 \mathrm{~h}$, following which $20 \mathrm{nM}$ miR-181a mimic, miR-181a inhibitor or irrelevant miRNA control (GenePharma, Shanghai, P.R. China) was added to a well. The cells were then transfected with Lipofectamine 2000 transfection reagent (Life Technologies, Thermo Fisher Scientific) according to the manufacturer's instructions for $4 \mathrm{~h}$ and then collected for further study.

\section{Extraction of RNA and miRNA and detection of cytokines and miR-181a}

The TRIzol reagent (Invitrogen, Thermo Fisher Scientific) method was used to extract total RNA of cells, serum and liver tissue according to the manufacturer's instructions. The Tiangen kit (TIANGEN Biotech Co., Beijing, P.R. China) was used to extract miRNA, and the SYBR Green Master Mix kit (Applied Biosystems, Thermo Fisher Scientific, Waltham, MA, USA) was used to determine the expression levels of miR-181a and cytokines TNF- $\alpha$, IL-4, IL-6, IL-10 and IL-1 $\beta$ (Table 1).

The cells, serum and liver tissue of mice and $M$. fortis were transferred into Eppendorf tubes (Eppendorf 
Table1 Primer names and primer sequence of quantitative PCR

\begin{tabular}{ll}
\hline Primer names & Primer sequence \\
\hline IL-4-F & GGTCTCAACCCCCAGCTAGT \\
IL-4-R & GCCGATGATCTCTCTCAAGTGAT \\
IL-6-F & CTGCAAGAGACTTCCATCCAG \\
IL-6-R & AGTGGTATAGACAGGTCTGTTGG \\
IL-10-F & CTTACTGACTGGCATGAGGATCA \\
IL-10-R & GCAGCTCTAGGAGCATGTGG \\
TNF-a-F & CCTGTAGCCCACGTCGTAG \\
TNF-a-R & GGGAGTAGACAAGGTACAACCC \\
GAPDH-F & AGGTCGGTGTGAACGGATTTG \\
GAPDH-R & TGTAGACCATGTAGTTGAGGTCA \\
U6-F & CTCGCTTCGGCAGCACA \\
U6-R & AACGCTTCACGAATTTGCGT \\
\hline
\end{tabular}

F, Forward; GAPDH, glyceraldehyde 3-phosphate dehydrogenase; R, reverse

AG, Hamburg, Germany). Lysate and miRNA Homogonote Addictive were added to the tubes and the contents mixed. Then, $300 \mu \mathrm{l}$ of acidic phenol was added to the tubes, and the contents were mixed to achieve separation. The liquid phase was transferred into a new tube, followed by the addition of anhydrous ethanol and mixing. Finally, miRNA wash solution was added into the filter column. After centrifugation, the contentration of miRNA was measured.

The PCR thermocycling program consisted of one cycle at $50{ }^{\circ} \mathrm{C}$ for $2 \mathrm{~min}$, one cycle at $90^{\circ} \mathrm{C}$ for $15 \mathrm{~s}$, followed by 40 cycles of $95^{\circ} \mathrm{C}$ for $5 \mathrm{~s}$ and $60^{\circ} \mathrm{C}$ for $34 \mathrm{~s}$, with the end time point of $60^{\circ} \mathrm{C}(34 \mathrm{~s})$ being the fluorescence signal detection point; each reaction was repeated three times. The quantitative PCR reaction (qPCR) was carried out according to the parameters specified in the Tiangen Extraction Kit (DP501; TIANGEN Biotech Co.); Applied Biosystems 7500 Real-Time PCR Software v2.0.5 was used for all calculations and analyses; GAPDH and U6 were used as the internal references for messenger RNA (mRNA) and miRNA quantification, respectively; and the relative expression of target genes was calculated by the $2^{-\Delta \Delta} \mathrm{Ct}$ method.

\section{Statistical analysis}

The experimental data were compared using SPSS version 22.0 statistical software (SPSS IBM Corp., Armonk, NY, USA). The t-test was used for two independent samples, and one-way analysis of variance and the least significant difference test was used for multiple groups. Significance was set at $P<0.05$, with $P<0.01$ indicating an extremely significant difference. All experiments were repeated a minimum of three separate times. GraphPad Prism 5 (GraphPad Software Inc., San Diego, CA, USA) was used for graphing the results.

\section{Results}

Analysis of miR-181a expression in the serum of mice and $M$. fortis infected with S. japonicum

As shown in Fig. 1, the expression of miR-181a in the serum of $M$. fortis infected with S. japonicum at 3, 7, 10 and $14 \mathrm{dpi}$, respectively was significantly lower than that in the uninfected control group at the same time points (Fig. 1a). In comparison, the expression level of miR-181a in mice infected with $S$. japonicum kept increasing over time and was significantly higher at 3, 7, 10 and $14 \mathrm{dpi}$, respectively, than that in the uninfected control group at the same time points (Fig. 1b).

\section{Analysis of miR-181a expression in the liver of mice and $M$. fortis infected with S. japonicum}

The livers of mice and M. fortis were collected separately, and total RNA was extracted to determine the changes in miR-181a expression. The results showed that the expression level of miR-181a in the livers of schistosome-infected mice at 3, 7, 10 and $14 \mathrm{dpi}$, respectively,
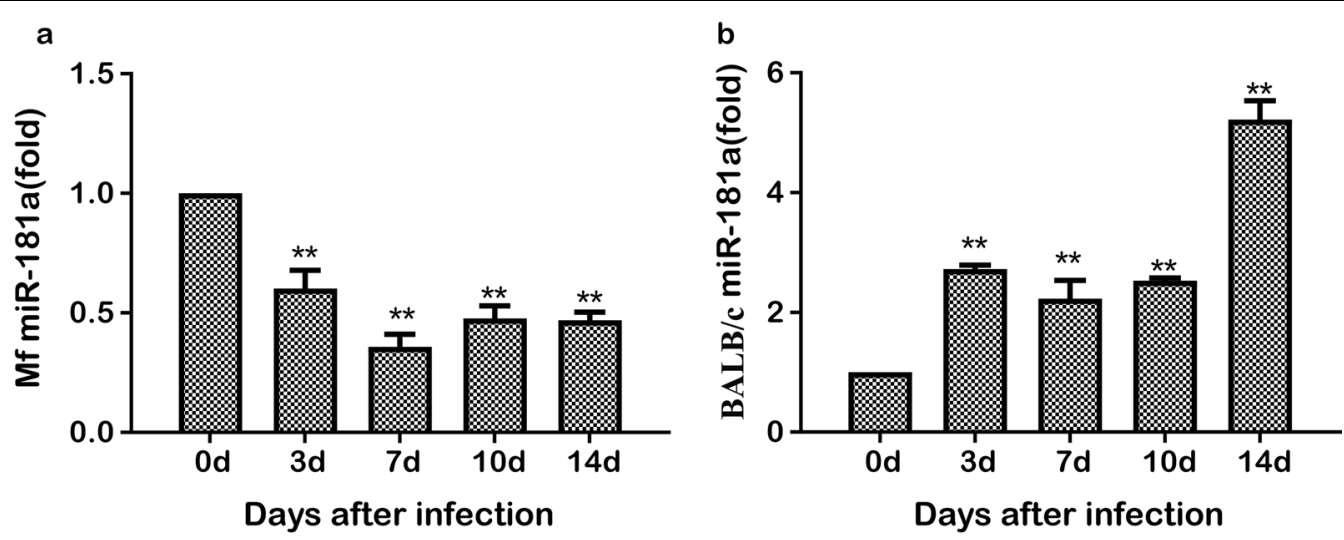

Fig. 1 Analysis of the expression level of miR-181a in the serum of male BALB/c mice (a) and reed vole Microtus fortis (b) infected with Schistosoma japonicum at different time periods post-infection. Asterisks indicate significant difference at ${ }^{*} P<0.05,{ }^{* *} P<0.01$. Abbreviations: MF, Microtus fortis 
was significantly higher than that in the uninfected control group at the same time points (Fig. 2a), while the expression level of miR-181a in the livers of schistosomeinfected $M$. fortis at 3, 7, 10 and $14 \mathrm{dpi}$, respectively, was significantly lower than that of the uninfected control group (Fig. 2b).
Analysis of changes in cytokine levels in mice and $M$. fortis at the early stage of schistosome infection

Changes in the expression of cytokines IL-10, IL-4, IL-6, IL-1 $\beta$ and TNF- $\alpha$ were measured in BALB/c mice, the susceptible host of $S$. japonicum, and in $M$. fortis, the resistant host, before infection and on 3, 7, 10 and 14 dpi, respectively. The results showed that the expression levels of anti-inflammatory factors IL-10 (Fig. 3a), IL-4 (Fig. 3b) and IL-6 (Fig. 3c) were higher in M. fortis than in mice and that the expression level of TNF- $\alpha$ (Fig. 3e)
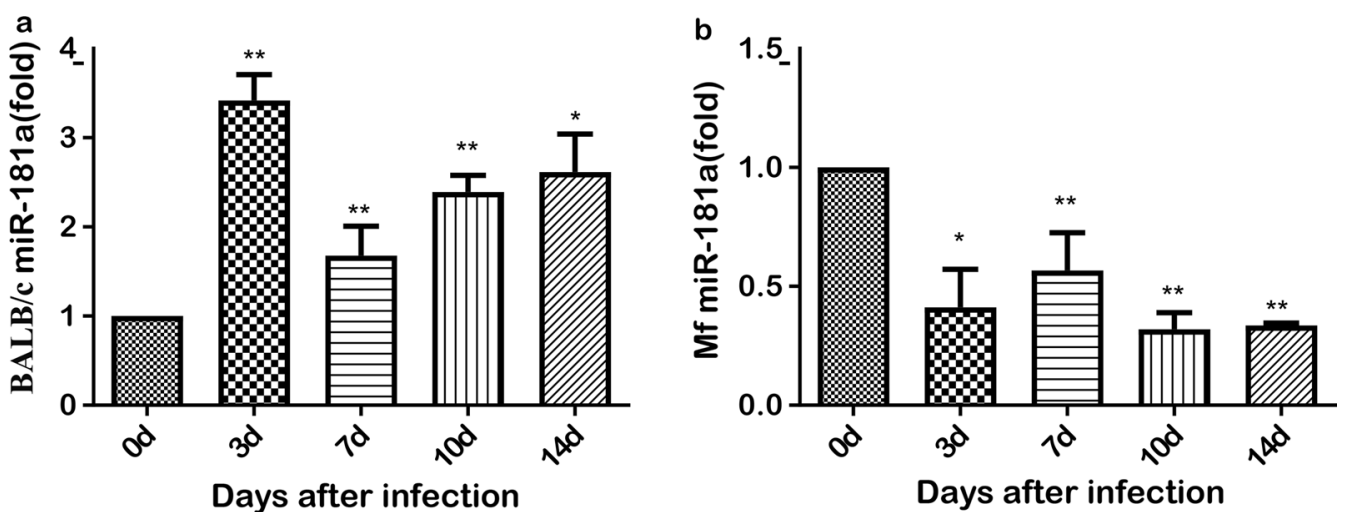

Fig. 2 Analysis of the expression level of miR-181a in the livers of male BALB/c mice (a) and M. fortis (b) infected with S. japonicum at different time points post-infection. Asterisks indicate significant difference at ${ }^{*} P<0.05,{ }^{* *} P<0.01$

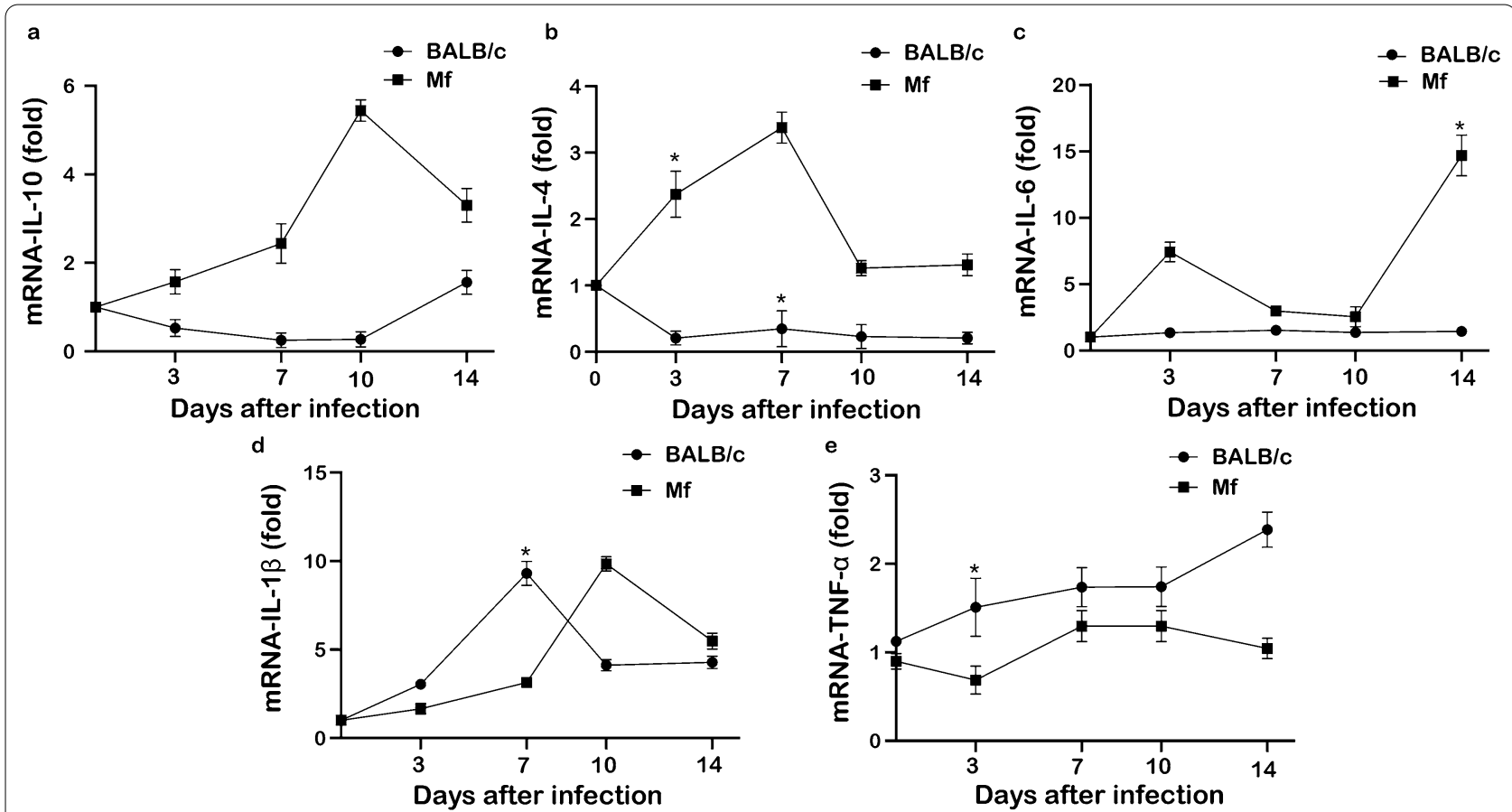

Fig. 3 Changes in the expression of IL-10 (a), IL-4 (b), IL-6 (c), IL-1 $\beta(\mathbf{d})$, and TNF- $\mathbf{a}(\mathbf{e})$ in mice and M. fortis after schistosome infection. Asterisk indicates significant difference at ${ }^{*} P<0.05$ 
was lower in $M$. fortis than in mice. The expression level of IL-1 $\beta$ (Fig. 3d) was higher in mice than in $M$. fortis on 3 and $7 \mathrm{dpi}$ and lower than that in M. fortis on 10 and $14 \mathrm{dpi}$. During the early stage of infection, the expression of the five detected cytokines, with the exception of IL-4, was generally upregulated post-schistosome infection in $M$. fortis. In mice, IL-10 and IL-4 expression was downregulated, IL- 6 expression was not significantly changed and TNF- $\alpha$ expression was upregulated and IL-1 $\beta$ expression was upregulated on 3 and $7 \mathrm{dpi}$ and downregulated on 10 and 14 dpi (see Fig. 3).

\section{Expression of miR-181a in TLR4-deficient mice}

TLR4-deficient mice and C57BL/6 mice (WT mice) were respectively infected with $S$. japonicum cercariae. Serum was collected 10 days later and the level of miR-181a was measured. The results showed that the level of miR-181a was significantly higher (3.8-fold) in TLR4-deficient mice than in WT mice and that the difference was highly significant (Fig. 4).

\section{Analysis of miR-181a and TLR4 expression after LPS stimulation of macrophages}

RAW264.7 macrophages were stimulated with LPS. Compared with the untreated blank control group, the expression of miR-181a was significantly downregulated in RAW264.7 macrophages after $12 \mathrm{~h}$ of LPS stimulation (about $40 \%$ of that of the control group) (Fig. 5a). The difference was very significant. In comparison, expression of TLR4 was upregulated to about 1.5-fold that of the control group; this difference was also very significant, as shown in Fig. 5b.

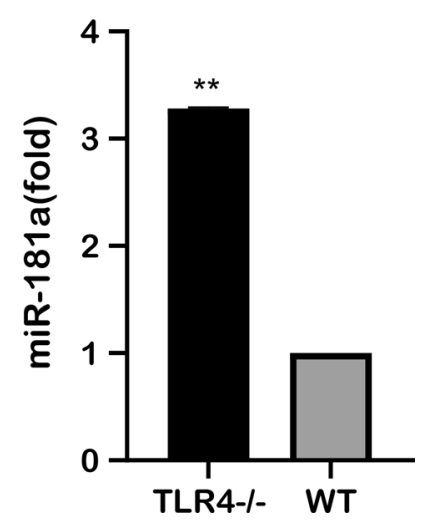

Fig. 4 Analysis of miR-181a in serum of TLR4-deficient mice and C57BL/6 mice 10 days after infection with S. japonicum. Asterisk indicates significant difference at ${ }^{*} P<0.01$. Abbreviation: TLR4, Toll-like receptor 4; WT wild type
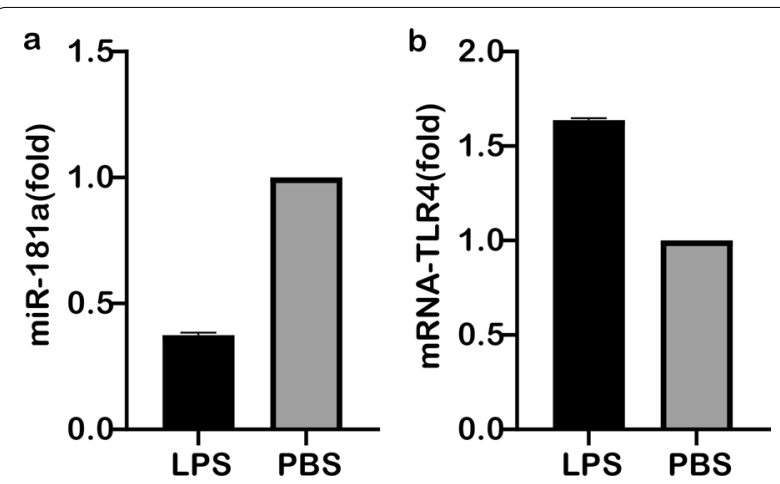

Fig. 5 Expression analysis of miR-181a (a) and TLR4 (b) after LPS stimulation of RAW264.7 macrophages. Abbreviation: PBS, Phosphate buffered saline

\section{Cytokine expression after LPS stimulation of RAW264.7 cells}

Following the stimulation of RAW264.7 cells with LPS, a TLR4 receptor ligand, the expression levels of IL-6, IL-1B and TNF- $\alpha$ in the cells were detected by qPCR. The results showed that the expression levels of IL-6 (Fig. 6a), IL-1 $\beta$ (Fig. 6b) and TNF- $\alpha$ (Fig. 6c) were significantly higher in RAW264.7 cells treated with LPS (by approx. 12.5-, 2.8- and 1.6-fold, respectively) than in the unstimulated control group and that the differences were all very significant.

\section{Analysis of miR-181a regulation on the expression of TLR4 receptor pathway inflammatory factors}

RAW264.7 cells were transfected with miR-181a mimic, miR-181a inhibitor and irrelevant microRNA, respectively, and then changes in the expression of cytokines IL-1 $\beta$ (Fig. 7a), TNF- $\alpha$ (Fig. 7b) and IL-6 (Fig. 7c) in the cells were detected. Comparison of the results with the non-transfection phosphate buffered saline control group showed that the expression of all three cytokines decreased by varying degrees after transfection with miR181a mimic and increased significantly in cells transfected with miR-181a inhibitor; in contrast, transfection with irrelevant control miRNA resulted in no significant difference in expression level (Fig. 7). These results suggest that miR-181a may play a negative regulatory role on the expression of these three Th1-type cytokines in RAW264.7 cells.

\section{Discussion}

Following the infection of $M$. fortis with S. japonicum, the development of schistosomes became stunted and abnormal, with most dying within 2 weeks after infection and only very few surviving until the third week post-infection. In this study, we used real-time qPCR 

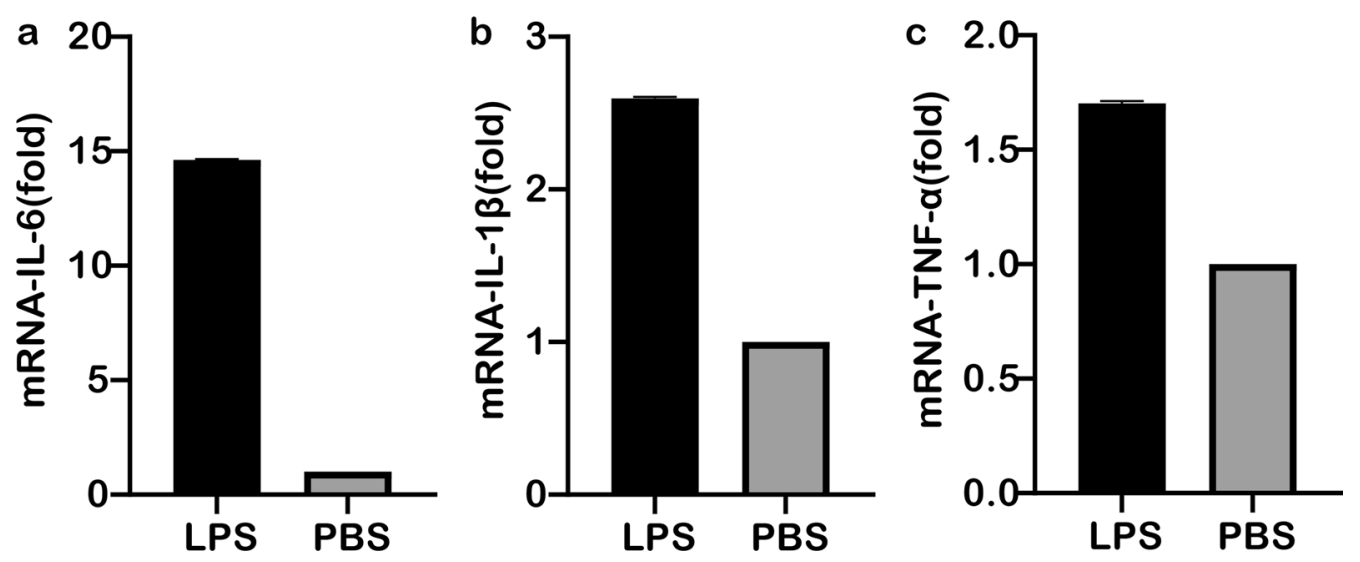

Fig. 6 The expression of IL-6 (a), IL-1 $\beta$ (b), TNF- $\alpha$ (c) after LPS stimulation of RAW264.7 macrophages
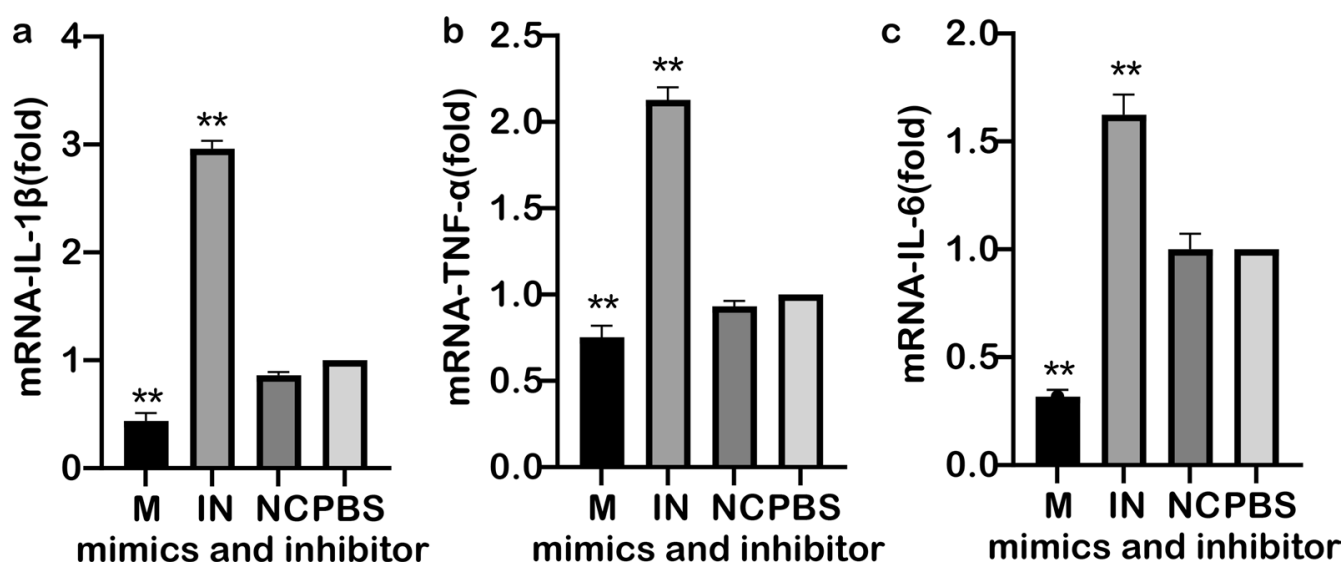

Fig. 7 Analysis of the expression of IL-1 $\beta$ (a), TNF- $-(\mathbf{b})$ and IL-6 (c) after transfection of RAW264.7 cells with miR-181a mimic and miR-181a inhibitor. Asterisks indicate significant different at ${ }^{* *} P<0.01$. Abbreviations: IN, Inhibitor; M, miR-181a mimic

technology to further analyze the difference in miR181a expression in the serum and liver of mice and $M$. fortis at different post-infection time points. The results showed that mir-181a expression was significantly upregulated and downregulated in the serum and liver of the schistosomiasis-infected mice and $M$. fortis respectively, at the different post-infection time points. Our recent study also showed that miR-181a negatively regulates the immune response of the schistosomulum antigen of $S$. japonicum, stimulates macrophages and promotes the conversion of macrophages to M2-type cells [20]. In the present study, we compared the expression levels of the Th1-type cytokines IL-1 $\beta$, TNF- $\alpha$ and IL- 6 and the Th2type cytokines IL-4 and IL-10 in susceptible host BALB/c mice and resistant host $M$. fortis on different days postinfection with $S$. japonicum. The results showed that during the early period of infection, the expression levels of the two Th2-type cytokines IL-10 and IL-4 were higher in $M$. fortis than in the BALB/c mice. Among the three Th1-type cytokines detected, the expression level of IL-6 was higher in $M$. fortis than in BALB/c mice, the expression level of TNF- $\alpha$ was lower in M. fortis than in BALB/c mice, and the expression level of IL-1 $\beta$ was lower in $M$. fortis than in BALB/c mice on 3 and $7 \mathrm{dpi}$ and higher in $M$. fortis than BALB/c mice on 10 and $14 \mathrm{dpi}$. At the same time, the expression of all five detected cytokines was generally upregulated at all post-inoculation measurement points in $M$. fortis compared with pre-infection levels. The expression of the two Th2-type cytokines IL-10 and IL-4 in mice was downregulated during the first 10 days post-infection. Among the three detected Th1 cytokines, the expression level of IL- 6 was not significantly changed, TNF- $\alpha$ was upregulated and IL- $1 \beta$ was upregulated on 3 and $7 \mathrm{dpi}$, and downregulated on 10 and $14 \mathrm{dpi}$. Taken together, these results further suggested that miR-181a played a key role in the early immune regulation of host resistance to schistosome infection. 
After the $S$. japonicum-susceptible host, namely the mice, were infected with schistosomes, upregulated expression of miR-181a may negatively regulate the host immunity against schistosome infection by downregulating the expression of Th2-type cytokines IL-10, IL-4, among others. In contrast, the downregulated expression of miR181a in the resistant host, namely $M$. fortis, may enhance the host immunity against schistosome infection by upregulating the expression of Th2-type cytokines IL-10 and Th1-type cytokines IL- $1 \beta$, TNF- $\alpha$ and IL- 6 . These may be some of the factors which affect the survival and development of schistosomes in these two hosts showing different susceptibility.

TLR4 is a crucial pathway which activates the NF-kB signaling pathway and plays an important role in the immune response of the host [21]. Research by Han Hongxiao and his colleagues revealed that some differential expression miRNAs between $S$. japonicumsusceptible host mice and the resistant host $M$. fortis may be related to the regulation of the TLR signaling pathway [22]. LPS is considered to be a bacterial endotoxin that stimulates the immune response of the host and acts mainly through activation of the TLR4 receptor pathway [23]. To understand the immunomodulatory mechanism of miR-181a, in the present study we transfected RAW264.7 cells with miR-181a mimic and inhibitor, respectively. The results showed that compared with the non-transfection control cells, the expression of three TLR4 pathway-related inflammatory factors, including IL- $1 \beta$, TNF- $\alpha$ and IL- 6 , decreased to varying degrees after transfection with miR-181a mimic, while the expression of these three cytokines was significantly increased in cells transfected with miR-181a inhibitor, indicating that miR-181a negatively regulated the expression of these three TLR4 pathway-related cytokines in RAW264.7 cells. The finding that the level of miR181a in serum was significantly higher in S. japonicuminfected TLR4-deficient mice than in WT mice further verified the correlation between miR-181a and the TLR4 pathway. After stimulation of RAW264.7 macrophages with LPS, a TLR4 receptor ligand, the expression of miR181a in the cells was significantly downregulated and the expression of TLR4 was upregulated. At the same time, the resultant expression of three TLR4 pathway-related cytokines, IL-1 $\beta$, TNF- $\alpha$ and IL-6, was significantly upregulated. These results suggested that there may be complex and subtle interactions between miR-181a and the TLR4 signaling pathway in S. japonicum infection. MiR-181a may regulate the expression of pro-inflammatory factors through the TLR4 receptor pathway and participate in the immune response against schistosome infection.

\section{Conclusions}

There may be mutual regulation between miR-181a and the TLR4 signaling pathway during S. japonicum infection. MiR-181a may regulate the expression of cytokines and other factors through the TLR4 receptor pathway and thus participate in the immunomodulatory effect of anti-S. japonicum infection.

\section{Abbreviations}

DMEM: Dulbecco's Modified Eagle's Medium; FBS: Fetal bovine serum; GMCSF: Granulocyte-macrophage colony-stimulating factor; IFN- $\gamma$ : Interferon- $\gamma$; IL: Interleukin; LPS: Lipopolysaccharide; miR-181a: MicroRNA-181a; miRNA: MicroRNA; TGF- $\beta$ : Transforming growth factor $\beta$; Th1: T-helper cell type 1 ; TNFa: Tumor necrosis factor $a$

\section{Acknowledgements}

The authors wish to thank the Shanghai Veterinary Research Institute for facilitating the implementation of this study.

\section{Authors' contributions}

TYX, LJ and HY conceived and designed the study, analyzed the data, drafted and critically revised the manuscript. TYX performed the experiment. ZQ, $Z Z H, S Y X, F Z Q, L K$ and LH helped in the implementation of the study and performed and interpreted the computational analysis. All authors read and approved the final manuscript.

\section{Funding}

This work was supported by the National Key Research and Development Program of China (2017YFD0501306) for Lin.

\section{Availability of data and materials}

The datasets used or analyzed during the current study are available from the corresponding author on reasonable request.

\section{Declarations}

\section{Ethics approval and consent to participate}

This study was carried out in strict accordance with the recommendations in the Animal Ethics Procedures and Guidelines of the People's Republic of China. The protocol was approved by the Committee on the Ethics of Animal Experiments of Shanghai Veterinary Research Institute, Chinese Academy of Agricultural Sciences (Protocol Number: SHVRI-SZ-20171013-06).

Consent for publication

Not applicable.

\section{Competing interests}

The authors declare that they have no competing interests.

\section{Author details}

${ }^{1}$ National Reference Laboratory for Animal Schistosomiasis, Shanghai Veterinary Research Institute, Chinese Academy of Agricultural Sciences, Shanghai 200241, P.R. China. ${ }^{2}$ Key Laboratory of Animal Parasitology of Ministry of Agriculture, Shanghai Veterinary Research Institute, Chinese Academy of Agricultural Sciences, Shanghai 200241, P.R. China.

Received: 22 June 2021 Accepted: 12 October 2021

Published online: 24 October 2021

\section{References}

1. McRae KM, Stear MJ, Good B, Keane OM. The host immune response to gastrointestinal nematode infection in sheep. Parasite Immunol. 2015:37:605-13. 
2. Chavez-Galan L, Olleros ML, Vesin D, Garcia I. Much more than M1 and M2 macrophages, there are also CD169(+) and TCR(+) macrophages. Front Immunol. 2015;6:263.

3. Rockey DC, Bissell DM. Noninvasive measures of liver fibrosis. Hepatology. 2006:43:2.

4. Anthony BJ, Ramm GA, McManus DP. Role of resident liver cells in the pathogenesis of schistosomiasis. Trends Parasitol. 2012;28:572-9.

5. Mantovani A, Biswas SK, Galdiero MR, Sica A, Locati M. Macrophage plasticity and polarization in tissue repair and remodelling. J Pathol. 2013;9(2):176-85

6. Takeda N, O'Dea EL, Doedens A, Kim JW, Weidemann A, Stockmann C, et al. Differential activation and antagonistic function of HIF-\{alpha\} isoforms in macrophages are essential for NO homeostasis. Genes Dev. 2010;24:491-501.

7. Weiskirchen R, Tacke F. Cellular and molecular functions of hepatic stellate cells in inflammatory responses and liver immunology. Hepatobiliary Surg Nutr. 2014;36:344-63.

8. He YX, Salafsky B, Ramaswamy K. Host-parasite relationships of Schistosoma japonicum in mammalian hosts. Trends Parasitol. 2001;17:320-4.

9. Li H, He YY, Lin JJ. The observation for the phenomenon of Microtus fortis aganisting Schistosoma japonicum. Chin J Vet Parasitol. 2000;8:12-5 (in Chinese).

10. Wu K. Schistosomiasis japonica among domestic and wild animals in China. Chin Vet J. 1957;3:98-100 (in Chinese)

11. Li SK, Zhu ZL, Jin BR, al. Uninfectibility to Schistosoma japonicum of Microtus fortis. Acta Parasitol Sin. 1965;2(1):103 (in Chinese).

12. Suryawanshi H, Lalwani MK, Ramasamy S, Rana R, Scaria V, Sivasubbu S, et al. Antagonism of microRNA function in zebrafish embryos by using locked nucleic acid enzymes (LNAzymes). ChemBioChem. 2012;13:584-9.

13. Okada H, Kohanbash G, Lotze MT. MicroRNAs in immune regulation-opportunities for cancer immunotherapy. Int J Biochem Cell Biol. 2010;42:1256-61.

14. Medzhitov R, Preston-Hurlburt P, Janeway CA Jr. A human homologue of the Drosophila Toll protein signals activation of adaptive immunity. Nature. 1997;388(66):394-7.
15. Janeway CA, Medzhitov R. Innate immune recognition. Annu Rev Immunol. 2002;20:197-216. https://doi.org/10.1146/annurev.immunol.20. 083001.084359 .

16. Ruprecht CR, Lanzavecchia A. Toll-like receptor stimulation as a third signal required for activation of human naive B cells. Eur J Immunol. 2006;36:810-6

17. Kuijper S, Turner CJ, Adams RH. Regulation of angiogenesis by Eph-ephrin interactions. Trends Cardiovasc Med. 2007;17:145-51.

18. Calvano JE, Agnese DM, Um JY, Goshima M, Singhal R, Coyle SM, et al. Modulation of the lipopolysaccharide receptor complex (CD14, TLR4, MD-2) and toll-like receptor 2 in systemic inflammatory response syndrome-positive patients with and without infection: relationship to tolerance. Shock. 2003;20(5):415-9.

19. Kumpf O, Hamann L, Schlag PM, Schumann RR. Pre- and postoperative cytokine release after in vitro whole blood lipopolysaccharide stimulation and frequent toll-like receptor 4 polymorphisms. Shock. 2006;25:123-8.

20. Tang $Y X$, Shen $Y X$, Hong $Y$, Zhai Q, Zhang ZH, Han HX, et al. MicroRNA181a plays a negative immune regulation role in macrophages stimulated with soluble schistosomula antigens of Schistosoma japonicum. Chin Vet Sci. 2018:48(02):204-10 (in Chinese).

21. Palsson-McDermott EM, O'Neill LA. Signal transduction by the lipopolysaccharide receptor, Toll-like receptor-4. Immunology. 2004;113(2):153-62

22. Yang J, Hong Y, Yuan C, Fu Z, Shi Y, Zhang M, et al. Microarray analysis of gene expression profiles of Schistosoma japonicum derived from less-susceptible host water buffalo and susceptible host goat. PLoS ONE. 2013:8(8): e70367.

23. Janardhan KS, Mclsaac M, Fowlie J, Shrivastav A, Caldwell S, Sharma RK, et al. Toll like receptor-4 expression in lipopolysaccharide induced lung inflammation. Histol Histopathol. 2006;7:687-96.

\section{Publisher's Note}

Springer Nature remains neutral with regard to jurisdictional claims in published maps and institutional affiliations.
Ready to submit your research? Choose BMC and benefit from:

- fast, convenient online submission

- thorough peer review by experienced researchers in your field

- rapid publication on acceptance

- support for research data, including large and complex data types

- gold Open Access which fosters wider collaboration and increased citations

- maximum visibility for your research: over $100 \mathrm{M}$ website views per year

At $\mathrm{BMC}$, research is always in progress.

Learn more biomedcentral.com/submissions 